\title{
Consensus
}

\section{Treatment of Hepatitis C virus infection in Italy: A consensus report from an expert panel}

\author{
Mauro Viganò ${ }^{A, *}$, Carlo Federico Perno ${ }^{\mathrm{B}}$, Antonio Craxì ${ }^{\mathrm{C}}$, \\ The AdHoc (Advancing Hepatitis C for the Optimization of Cure) Working Party \\ A Hepatology Unit, Ospedale San Giuseppe, University of Milan, Milan, Italy \\ B Virology Unit, Department of Experimental Medicine and Surgery, "Tor Vergata" University of Rome, Rome, Italy \\ C Department of Gastroenterology, DiBiMIS, University of Palermo, Palermo, Italy
}

\section{A R T I C L E I N F O}

\section{Article history:}

Received 8 March 2017

Accepted 31 March 2017

Available online 12 April 2017

\section{Keywords:}

Antiviral treatment

Cirrhosis

Direct-acting antiviral agents

Hepatitis C

RAS

Treatment failure

\begin{abstract}
A B S T R A C T
Hepatitis $\mathrm{C}$ virus (HCV) infection remains one of the main causes of chronic liver disease worldwide. The advent of direct-acting antivirals (DAAs) has significantly improved the course of patients with chronic HCV infection ( $\mathrm{CHC}$ ), due to the ability of these drugs to achieve high rates of sustained virological response (SVR). These exceedingly high rates of SVR and the excellent safety data have been confirmed in real life practice. Evolving guidelines have been issued by national and international scientific societies in accordance with the progression of clinical knowledge and the availability of new DAAs. These recommendations, however, may not be applied universally because of delays in drugs reimbursability in different countries and because some National Health Systems identify only patients with advanced disease as a treatment priority. Italy in this regard is a prototype about DAAs treatment of CHC patients.

With the aim to assess the Italian treatment experience with DAAs and to respond to unmet needs in treatment optimization of antiviral therapy in specific settings of $\mathrm{CHC}$ patients, a group of Italian experts met in Stresa in February 2017. The summary of the considerations arising from this two-day meeting and some statements regarding a few open issues are reported in this position paper.
\end{abstract}

(c) 2017 Editrice Gastroenterologica Italiana S.r.l. Published by Elsevier Ltd. All rights reserved.

\section{The HCV treatment scenario in Italy}

Hepatitis $\mathrm{C}$ virus (HCV) infection is one of the main causes of chronic liver disease worldwide and is responsible for a large proportion of liver-related deaths, mostly because of hepatocellular carcinoma (HCC) and cirrhosis [1,2].

Approximately 180 million people worldwide are currently infected with HCV, although reliable epidemiological data are elusive because of the asymptomatic nature of the infection and the

Alessio Aghemo ${ }^{\mathrm{a}}$, Alfredo Alberti ${ }^{\mathrm{b}}$, Pietro Andreone ${ }^{\mathrm{c}}$, Massimo Andreoni ${ }^{\mathrm{d}}$, Stefano Bonora $^{\mathrm{e}}$, Maurizia Rossana Brunetto ${ }^{\mathrm{f}}$, Raffaele Bruno ${ }^{\mathrm{g}}$, Savino Bruno ${ }^{\mathrm{h}}$, Vincenza Calvaruso $^{\mathrm{i}}$, Nicola Caporaso ${ }^{\mathrm{j}}$, Francesca Ceccherini-Silberstein ${ }^{\mathrm{k}}$, Valeria Cento ${ }^{\mathrm{k}}$, Alessia Ciancio ${ }^{1}$, Piero Colombatto ${ }^{\mathrm{f}}$, Elisabetta Degasperi ${ }^{\mathrm{a}}$, Vito Di Marco ${ }^{\mathrm{i}}$, Giovanni Di Perri ${ }^{\mathrm{e}}$, Gianpiero D’offizi ${ }^{\mathrm{m}}$, Stefano Fagiuoli ${ }^{\mathrm{n}}$, Carlo Ferrari ${ }^{\circ}$, Giovanni Battista Gaeta $^{\mathrm{p}}$, Adriano Pellicelli ${ }^{\mathrm{q}}$, Salvatore Petta ${ }^{\mathrm{i}}$, Sara Piovesan ${ }^{\mathrm{b}}$, Massimo Puoti ${ }^{\mathrm{r}}$, Giovanni Raimondo ${ }^{\mathrm{s}}$, Francesco Paolo Russo ${ }^{\mathrm{t}}$, Gloria Taliani ${ }^{\mathrm{u}}$, Ugo Trama ${ }^{\mathrm{v}}$, Erica Villa ${ }^{\mathrm{w}}$, Anna Linda Zignego ${ }^{x}$

$\mathrm{a}-\mathrm{x}$ See Appendix A.

* Corresponding author at: Hepatology Unit, Ospedale San Giuseppe, University of Milan, Via S. Vittore 12, 20123 Milan, Italy. Fax: +39 285994267.

E-mail address: mvigano72@gmail.com (M. Viganò). lack of screening programs in most countries [3,4]. Even in Italy the exact number of HCV-positive patients is unknown, however it is believed that there is a large proportion of $\mathrm{CHC}$ patients (roughly $1 \%$ ), most with age $>60$ years with advanced liver disease, with nearly 80,000 cirrhotics and at least 3000 with decompensated disease. On the other hand a reliable assessment of the impact of the infection is represented by the number of patients transplanted due to HCV (500/year) and by deaths caused by the virus (about $10,000 /$ year).

Until the end of February 2017, 68,270 HCV patients had been treated with DAAs (http://www.agenziafarmaco.gov.it/content/ aggiornamento-epatite-c). According to the AIFA rules only patients with most advanced liver disease, cured HCC, listed for liver transplant (LT), with HCV recurrence after LT or after transplant of solid organ other than liver, and with severe HCV-related extra-hepatic manifestations (EHMs) could be considered for the DAAs treatment. The treated patients were: 43,453 (64\%) cirrhotics with or without HCC (cured with surgical or loco-regional therapies); 18,497 (27\%) METAVIR fibrosis score F3; 3191 (4\%) with severe EHMs, i.e. cryoglobulinemic syndrome with organ damage or B-cell lymphoproliferative syndromes; 1908 (3\%) with post-LT recurrence, 620 (1\%) METAVIR F0-F2 who underwent 
pegylated interferon (Peg-IFN) + Ribavirin (Rbv) + simeprevir (SIM) treatment; $309(0,5 \%)$ listed for LT with cirrhosis MELD <25 and/or HCC within the Milan criteria with the possibility of waiting in a list of at least 2 months and $292(0.5 \%)$ with CHC and non liver solid organ transplantation or bone marrow transplantation.

Based on the Italian National Health System the reimbursability of DAAs was therefore granted according to urgency of treatment, without the possibility to use these drugs in patients with less advanced fibrosis (F0-F2). Moreover the price of DAAs was negotiated centrally with price/volume agreements and pay-per-patient reimbursement, regardless of treatment duration with a cost per patient of nearly 6000-10,000 euro. These methods of reimbursement have allowed using DAAs longer without the need to identify the patients being treated for shorter time to save costs.

\section{The near future scenario of HCV treatment}

Although in Italy the treatment was initially reserved exclusively to patients with advanced liver disease, in the last months of 2016 the percentage of F3 patients undergoing DAAs gradually increased up to reaching the number of F4 patients, to indicate that treatment indication is moving toward lesser stages of disease. Recently it has been estimated that are still present at least $100,000-150,000$ F0-F3 and 10,000 F4 patients that need antiviral treatment. These patients in the next four years might all be treated with an annual cost of 400 million euros. The progressive decline of patients with urgency of treatment will allow in the short term to expand treatment criteria without, however, bear the cure for all infected patients. Unfortunately our country lacks of a plan for universal or targeted screening of infected patients; as well as of a strategy for retreatment of patients who previously failed DAAs, and a wide access to DAAs treatment for people at high risk of viral transmission (i.e. women of childbearing age who wish to become pregnant; healthcare professional; people with sexually transmitted diseases; prisoners; people who inject drugs).

Treatment of $\mathrm{CHC}$ has been revolutionized in the last few years by the introduction of highly effective and well tolerated DAAs able to achieve $>90 \%$ rates of SVR in many groups of patients including those with more advanced liver disease. Successful anti-HCV treatment can stop liver disease progression eventually reducing both liver-related and overall mortality [5,6].

The second wave of DAAs has significantly improved SVR rates, moreover these drugs are better tolerated and have more convenient once-daily dosing regimens with shorter treatment schedules, i.e. 12 weeks in the majority of patients and in selected ones only 8 weeks.

The future combination regimens with new generation oral agents may further increase SVR rates without the need for Rbv, with shorter treatment durations and with more favorable tolerability profiles. With these treatments we might finally have the potential to treat the infection and not just the disease, with the aim to eradicate HCV worldwide.

However, also with these new DAAs some types of patients will remain "difficult to treat", such as: DAAs-experienced patients with resistance associated variants (RAVs); patients with decompensated cirrhosis, and genotype 3 cirrhotic patients.

During the last AASLD 2016 meeting, several new DAAs regimens were presented. The combination of sofosbuvir (SOF)/velpatasvir (VEL) with the new pangenotypic HCV NS3/4A protease inhibitor (PI) Voxilaprevir (VOX) is one of the new a fixed-dose formulations. The POLARIS-2 study compared treatment with SOF/VEL/VOX for 8 weeks to SOF/VEL for 12 weeks in genotype 1-6 patients with and without compensated cirrhosis who have not previously received treatment with DAAs. Patients with genotype 3 infection and cirrhosis were excluded from this study, but included in the POLARIS-3 study. Eight weeks of SOF/VEL/VOX achieved 95\% SVR rates compared to 98\% with the 12 week SOF/VEL regimen. The SVR rates with SOF/VEL/VOX and SOF/VEL were $91 \%$ vs $99 \%$ and $96 \%$ vs $98 \%$ in patients with and without cirrhosis, respectively. Higher relapse rates were reported in the 8 weeks treatment arm particularly in genotype 1a patients [7]. POLARIS-4 study compared 12 weeks of SOF/VEL/VOX with SOF/VEL in 182 and 151 patients, respectively, with previous failure of non-NS5A containing DAA regimens. SVR rates were higher among patients treated with SOF/VEL/VOX compared to SOF/VEL (97\% vs 90\%) [8].

In the randomized, open label, multicenter ENDURANCE-1 study, 703 naïve or treatment-experienced (prior IFN \pm Rbv or $\mathrm{SOF}+\mathrm{Rbv} \pm \mathrm{IFN}$ ) genotype 1 patients without cirrhosis or with HIV co-infection were treated for either 8 or 12 weeks with the combination of pangenotipic NS3/4A PI Glecaprevir (GLE) and pangenotipic NS5A inhibitor Pibrentasvir (PIB) [9]. Both arms showed high rates of SVR: $99 \%$ and $100 \%$, respectively. The same high SVR rate (99\%) was also reported in the ENDURANCE-4 trial, that investigated the safety and efficacy of a 12-week GLE/PIB treatment in 121 non cirrhotic patients infected with HCV genotype 4-6, either naïve or treatment-experienced (prior IFN \pm Rbv or SOF + Rbv \pm IFN) [10], and also in the ENDURANCE-2 trial, that investigated the safety and efficacy of a 12-week GLE/PIB treatment in 302 naïve or treatment-experienced (prior IFN \pm Rbv or $\mathrm{SOF}+\mathrm{Rbv} \pm$ IFN) non cirrhotic genotype 2 patients [11].

The SURVEYOR-II, Part 3 study evaluated the efficacy and safety of GLE/PIB for 12 or 16 weeks in 131 CHC patients genotype 3 with or without cirrhosis including those with prior treatmentexperience [12]. In experienced cirrhotic patients, 16 weeks of GLE/PIB achieved $96 \%$ of SVR compared to $98 \%$ of naïve patients treated for 12 weeks, whereas in treatment-experienced non cirrhotic patients, 16 weeks achieved $96 \%$ of SVR vs $91 \%$ in those treated for 12 weeks.

The EXPEDITION-IV study reported 98\% SVR rate in 104 genotype 1-6 patients with severe renal impairment (82\% on hemodialysis) treated with 12 weeks of GLE/PIB [13]. This is a promising study, since the DAAs recommended to date for treatment of $\mathrm{HCV}$ in patients with advanced renal insufficiency are predominantly active in genotypes 1 and 4, whereas genotypes 2 and 3 patients with end-stage renal disease have little chance of treatment.

An interesting new fixed-dose triplet is the pangenotypic MK3 combination, that includes the NS5B polymerase nucleotide inhibitor uprifosbuvir (formerly MK-3682), the already-registered NS3/4A PI Grazoprevir (GZR) and the next-generation NS5A inhibitor Ruzasvir (RZR). The C-CREST trial evaluated the safety and efficacy of the MK3 regimen, with or without Rbv, in 664 genotype 1-3 patients with and without cirrhosis [14]. Genotype 1 patients were randomized to 8 or 12 weeks of the triplet; genotype 2 treatment-naïve patients were randomized to 8 or 12 weeks of the triplet, with or without Rbv, and compared with 16 weeks of only MK3; genotype 3 treatment-naïve and experienced patients were randomized to 8 vs 12 vs 16 weeks, with or without Rbv. MK3 for 8 or 12 weeks achieved 93\% vs 98\% SVR rates in genotype 1a, and $98 \%$ vs $100 \%$ SVR rate in genotype $1 \mathrm{~b}$ patients. In genotype 2 treatment-naïve patients, SVR rates were $86 \%, 97 \%$ and $100 \%$ in the 8,12 and 16 weeks arms, respectively. In genotype 3, MK3 regimen showed $95 \%, 97 \%$ and $96 \%$ of SVR in the 8,12 or 16 weeks arms of treatment. The C-SURGE study evaluated the safety and efficacy of the MK3 regimen in patients who relapsed after LDV/SOF or elbasvir (EBR)/GZR treatment. Eight weeks after the end of treatment, the SVR rates were $98 \%$ and $100 \%$ in patients randomized to receive 16 weeks MK3 + Rbv or 24 weeks of MK3 without Rbv, respectively [15]. 


\section{Treatment of patients with genotype $2 \mathrm{HCV}$ infection}

\subsection{Genotype 2 non cirrhotic and compensated cirrhotic patients}

According to current EASL Guidelines, genotype 2 patients should be treated with the combination of the NS5B inhibitor SOF plus the NS5A inhibitor Daclatasvir (DCV) or VEL: the recommended treatment schedule is SOF+DCV or SOF/VEL for 12 weeks in non cirrhotic and compensated cirrhotic patients (Child Pugh score A), independently from previous treatment history [16]. Although DCV is active against HCV genotype 2, literature data concerning SOF + DCV treatment in genotype 2 patients are limited: indeed SOF + DCV has been evaluated in a Phase II study in 26 genotype 2 naïve patients, including only 6 (23\%) patients with cirrhosis. The combination of SOF $+\mathrm{DCV} \pm \mathrm{Rbv}$ for 24 weeks resulted in 24/26 (92\%) patients achieving SVR [17]. The following Phase III ALLY-1 trial evaluated SOF + DCV treatment in patients with advanced cirrhosis; including a small number of genotype 2 infected patients: overall 4 out of 5 patients (80\%) achieved SVR [18]. A recent subanalysis of 36 genotype 2 patients, enrolled in 5 Phase II and III clinical trials with SOF+DCV-based regimens, reported 97\% SVR rates, confirming optimal efficacy of this regimen in such patients, where presence of baseline RAV to NS5A did not affect the achievement of SVR [19]. Efficacy of SOF + DCV combination in genotype 2 patients has been confirmed also by real-life data: a small Italian study in 19 genotype 2 patients evaluating SOF + DCV for 12 or 24 weeks reported $100 \%$ SVR rates, where 11 out of the 19 patients had cirrhosis and history of previous treatment failure, and 5 patients had decompensated cirrhosis [20]. Recently, the RESIST-HCV (Rete Sicilia Selezione Terapia-HCV) reported high rate of SVR (90\%) in Child-A genotype 2 cirrhotic patients treated for 12 weeks with SOF + DCV [21].

Treatment of genotype 2 patients with SOF/VEL has been evaluated in the ASTRAL- 1 and 2 trials, where respectively 104/104 (100\%) and 133/134 (99\%) patients achieved SVR following 12 weeks of SOF/VEL fixed dose combination. Patient population in both ASTRAL trials included approximately $15 \%$ of compensated cirrhotic patients $[22,23]$. Latest EASL treatment guidelines define $\mathrm{SOF}+\mathrm{Rbv}$ combination as suboptimal for genotype 2 patients. This statement comes from real-life reports showing SVR rates below $90 \%$, especially in treatment experienced and cirrhotic patients receiving SOF + Rbv for 12 weeks [24]. These data were also confirmed by a sub-analysis of genotype 2 patients treated in the TARGET study, where 49/62 (79\%) and 20/24 (83\%) cirrhotic patients achieved SVR following a 12 or 16 -week course of SOF+ Rbv, compared with 201/221 (91\%) and 13/14 (93\%) non cirrhotic patients, respectively. Baseline Rbv dose was significantly associated with the probability of achieving SVR, where optimal chances for SVR were predicted by baseline Rbv dose $\geq 15 \mathrm{mg} / \mathrm{kg}$ [25]. In addition to suboptimal efficacy of SOF + Rbv in patients with advanced fibrosis, the possibility to consider SOF + Rbv as a potential option for selected patients (naïve, no cirrhosis), in order to reduce costs deriving from multiple DAAs use, has to be discouraged as well, because indirect treatment costs would increase due to management of Rbv-related side effects.

\subsection{Genotype 2 patients with decompensated cirrhosis}

In patients with decompensated cirrhosis (Child Pugh B/C), EASL guidelines recommend SOF + DCV or SOF/VEL + Rbv for 12 weeks, however treatment extension to 24 weeks should be considered.

The ASTRAL-4 study compared SOF/VEL \pm Rbv for 12 weeks vs SOF/VEL for 24 weeks in patients with decompensated cirrhosis: overall 12 genotype 2 patients were included in the study and 11 achieved SVR except for one patient who died because of liver failure one month after starting treatment [26].
3.3. Retreatment of genotype 2 patients with failure to NS5A-containing regimens

Genotype 2 patients failing to achieve SVR following SOF + Rbv can be retreated with $\mathrm{SOF}+\mathrm{DCV}+\mathrm{Rbv}$ or $\mathrm{SOF} / \mathrm{VEL}+\mathrm{Rbv}$ for 12 weeks (non cirrhotic) or 24 weeks (advanced fibrosis F3/4). SOF/VEL combination in 33 genotype 2 SOF-experienced patients achieved 97\% SVR rate in POLARIS-4 trial, where cirrhosis accounted for $46 \%$ of patient population [8]. Future retreatment options include the SOF/VEL/VOX fixed dose combination that achieved 100\% SVR in 33 genotype 2 patients with previous treatment failure to SOF-based regimens in the POLARIS- 4 study.

While waiting for future retreatment options, viral sequencing for confirmation of HCV genotype and RAV assessment is recommended in order to individualize and improve retreatment strategy. Indeed, a recombinant $\mathrm{HCV}-2 \mathrm{k} / 1 \mathrm{~b}$ strain carrying the $5^{\prime}$ HCV-2 and 3' HCV-1 region has been described in literature, especially in Eastern Europe [27]: these patients could be misclassified as HCV genotype 2 according to commercial assays for HCV genotype evaluating the $5^{\prime}$ UTR region, and consequently they could potentially have been undertreated with a genotype 2 schedule not suitable for genotype 1 . Assessment of HCV genotype by viral sequencing could identify the recombinant strain and optimize retreatment option, although this issue could also be solved by upcoming availability of pan-genotypic regimens.

Resistance analysis in genotype 2 patients failing to achieve the SVR to NS5B-containing regimens found no NS5B RAV development, as expected by the low replicative fitness exhibited by viral strains carrying amminoacidic changes in the NS5B polymerase domain [28]. In addition, presence of NS5A RAV at baseline seems not to affect efficacy of SOF + DCV regimen in genotype 2 patients, as demonstrated by subanalysis of DCV clinical trials [19]. However, till the availability of next generation drugs, Rbv addition is still recommended in order to optimize retreatment response rates, as Rbv has been shown to improve viral clearance, especially in patients with advanced fibrosis and baseline RAVs.

\subsection{Statements}

- SOF+ DCV or SOF/VEL for 12 weeks are the standard of care treatment for genotype 2 F0-F4 patients (Child Pugh A).

- In genotype 2 patients with decompensated cirrhosis (Child Pugh $\mathrm{B} / \mathrm{C}), \mathrm{SOF}+\mathrm{DCV} \pm \mathrm{Rbv}$ or $\mathrm{SOF} / \mathrm{VEL} \pm \mathrm{Rbv}$ for 24 weeks is recommended.

- SOF + Rbv has suboptimal efficacy in genotype 2 cirrhotic patients and is not recommended as well as in the other patient groups due to Rbv-related side effects.

- Genotype 2 patients failing to achieve the SVR using SOF+Rbv regimen should be retreated according to EASL recommendations. Viral sequencing to confirm HCV genotype and RAV analysis is suggested to optimize retreatment strategies, while Rbv addition is a valid option till availability of next-generation drugs.

\section{Treatment of patients with genotype $3 \mathrm{HCV}$ infection}

Current treatment recommendations in genotype 3 infected patients differ according to liver fibrosis stage and previous treatment experience.

\subsection{Genotype 3 patients without cirrhosis}

According to American (AASLD) Guidelines, all genotype 3 patients without cirrhosis should be treated with SOF+DCV or SOF/VEL for 12 weeks, while EASL Guidelines recommend Rbv addition in genotype 3 non cirrhotic patients with previous treatment 
failure to Peg-IFN + Rbv therapy and baseline NS5A RAS Y93H (if resistance testing available) $[16,29]$.

In the ALLY-3 trial, 73/75 (97\%) naïve and 32/34 (94\%) treatment experienced non cirrhotic patients achieved SVR following $\mathrm{SOF}+\mathrm{DCV}$ administration for 12 weeks [30]. Addition of Rbv is recommended, as SVR decreased to $50 \%$ in patients with baseline $Y 93 \mathrm{H}$ [31].

Efficacy of SOF/VEL combination in genotype 3 patients has been investigated in the ASTRAL-3 trial, where 160/163 (98\%) naive and 31/34 (91\%) experienced patients without cirrhosis achieved SVR following 12 weeks of SOF/VEL treatment. SVR rate in patients with no NS5A RAV at baseline was $97 \%$ (225/231) vs $88 \%(38 / 43)$ in patients with baseline NS5A RAVs (16\% prevalence) and $84 \%$ $(21 / 25)$ in patients with baseline $\mathrm{Y93H}$ [23]. Eighty-nine genotype 3 patients received SOF/VEL combination for 12 weeks also in the POLARIS-2 trial, resulting in 86 patients (97\%) achieving SVR [7].

\subsection{Genotype 3 patients with cirrhosis}

In patients with cirrhosis, European and American Guidelines recommend SOF + DCV + Rbv for 24 weeks or SOF/VEL + Rbv for 12 weeks independently from previous treatment experience. Rbv should be added to SOF/VEL in presence of baseline NS5A RAVY93H, while treatment should be extended to 24 weeks if Rbv cannot be administered [16,29].

The SOF + DCV + Rbv 24-week course was recommended basing on expert opinions, as no data has been generated yet by clinical trials in cirrhotic patients with this treatment schedule. Indeed Phase III trials investigated only 12 or 16 week-courses of SOF+DCV. The ALLY-3 trial demonstrated suboptimal efficacy of SOF +DCV for 12 weeks in cirrhotic patients, as only 11/19 (58\%) naive and 9/13 (69\%) experienced patients achieved SVR, where high relapse rate was probably related to the study design, not including Rbv administration [30]. Addition of Rbv and treatment extension to 16 weeks explored by the ALLY-3+ trial resulted in 21/24 (88\%) and $24 / 26$ (92\%) patients achieving SVR following 12 or 16 weeks of SOF+DCV, where Peg-IFN-experienced patients accounted for the majority of patient population [32]. Despite the absence of clinical trial data, efficacy of SOF + DCV + Rbv for 24 weeks has been confirmed in real-life settings. The RESIST-HCV reported 91\% SVR rate in Child-A genotype 3 cirrhotic patients treated for 24 weeks with SOF + DCV + Rbv but only $84 \%$ in those treated without Rbv [21]. The Lombardia Hepatitis Network reported overall 97\% SVR in genotype 3 patients with advanced fibrosis (F3/4) [33]. Excellent result were also reported by the CLEO, showing $98 \%$ and $96 \%$ SVR rates in patients treated with 24 weeks of SOF + DCV + Rbv flat dose $(800 \mathrm{mg})$ or SOF+DCV-weight-based Rbv dose, respectively [34]. The French compassionate use program in genotype 3 cirrhotic patients treated with 24 weeks of SOF + DCV reported 86\% SVR rate in patients with any degree of cirrhosis without SVR increase in those who received additional Rbv (82\%) [35].

In the ASTRAL-3 trial, 40/43 (93\%) naive and 33/37 (89\%) experienced genotype 3 patients with cirrhosis achieved SVR following SOF/VEL for 12 weeks [23]. In the POLARIS-3 trial, 109 genotype 3 cirrhotic patients, including 32 (29\%) with previous PegIFN + Rbv treatment failure, received SOF/VEL for 12 weeks with $98 \%$ (105/109) SVR rate [36].

\subsection{Genotype 3 patients with decompensated cirrhosis}

Patients with decompensated cirrhosis should receive $\mathrm{SOF}+\mathrm{DCV}+\mathrm{Rbv}$ for 24 weeks or SOF/VEL combination for 24 weeks according to EASL guidelines [16].

In a large European compassionate use program the use of 24 weeks of $\mathrm{SOF}+\mathrm{DCV} \pm \mathrm{Rbv}$ in Child $\mathrm{B} / \mathrm{C} \mathrm{CHC}$ patients, reported
$79 \%(19 / 24)$ and $88 \%(15 / 17)$ SVR rates in patients treated with $\mathrm{SOF}+\mathrm{DCV}$ and SOF + DCV + Rbv, respetively [37].

The ASTRAL-4 trial, comparing SOF/VEL with or without Rbv for 12 weeks vs SOF/VEL for 24 weeks in 36 genotype 3 decompensated patients, showed the best SVR rates in the 12 -week SOF/VEL + Rbv arm: $11 / 13(85 \%)$ patients achieved SVR compared to $7 / 14(50 \%)$ and 6/12 (50\%) patients in the SOF/VEL 12 and 24 week course, respectively [26].

\subsection{Future treatment options for naive and Peg-IFN experienced genotype 3 patients}

All upcoming second generation DAA combinations are effective in genotype 3 patients and have already provided very promising results: the GLE/PIB combination was evaluated in genotype 3 naive non cirrhotic patients with a shortened 8-week treatment course in the SURVEYOR-II (Part 2) and ENDURANCE-3 trials, where 28/29 (97\%) and 149/157 (95\%) patients achieved SVR, respectively [38,39]. The SURVEYOR-II (Part 3) investigated the efficacy of GLE/PIB for 12 or 16 weeks in 131 genotype 3 patients with previous treatment experience (19\% failing SOF + Rbv combination) and/or cirrhosis, providing SVR rates exceeding $90 \%$ across all treatment arms [12]. As GLE/PIB combination shows no renal metabolism, it represents a promising treatment option for genotype 3 patients with advanced chronic kidney disease, who currently cannot be treated with SOF-based combinations.

The MK3 triple combination was evaluated in the C-CREST program, where 337 genotype 3 patients received MK3 \pm Rbv for 8 , 12 or 16 weeks. SVR rates were $95 \%$ in the 8 -week arm, where $74 / 103$ (72\%) patients were treatment-naive and only non cirrhotic patients were included. In the 12-week arm, 155/159(97\%) patients achieved SVR, this arm including also patients with cirrhosis and 58 (36\%) Peg-IFN + Rbv experienced patients. In the 16-week arm, $72 / 75$ (96\%) achieved SVR, where the majority of patients $(61 / 75$, $81 \%$ ) were treatment experienced. Overall SVR rates in treatment experienced patients with cirrhosis exceeded $96 \%$ both in the 12 and 16-week duration, independently from Rbv addition [14]. The shortened 8-week treatment duration in patients with cirrhosis has been evaluated for the first time with the triple SOF/VEL/VOX pan-genotypic combination, where the POLARIS-3 trial showed $96 \%$ SVR rate in 110 genotype 3 patients, including 35 (32\%) Peg-IFNexperienced patients [36].

\subsection{Retreatment of genotype 3 patients with failure to NS5A-containing regimens}

Virologic failure to a NS5A-containing regimen, i.e. DCV in genotype 3 patients, results in selection of NS5A RAV, which show high replicative fitness compared to wild-type virus and consequently persist long-term, exhibiting also cross-resistance with other NS5A inhibitors. Retreatment options for genotype 3 patients failing SOF + DCV should be based on a DAA combination regimen including all three NS3, NS5A and NS5B inhibitors. The POLARIS-1 trial showed very promising result of SOF/VEL/VOX combination in genotype 3 NS5A-experienced patients: 74/78 (95\%) patients achieved SVR, while baseline presence of NS5A RAV did not affect treatment efficacy [40].

\subsection{Statements}

- Genotype 3 patients without cirrhosis should receive SOF + DCV or SOF/VEL for 12 weeks if treatment naive, while Rbv should be added in Peg-IFN experienced patients in presence of baseline NS5A RAV Y93H. 
- Genotype 3 patients with compensated cirrhosis should be treated with $\mathrm{SOF}+\mathrm{DCV}+\mathrm{Rbv}$ for 24 weeks or SOF/VEL + Rbv (if baseline NS5A RAV Y93H is present or documented) for 12 weeks.

- In genotype 3 patients with decompensated cirrhosis, $\mathrm{SOF} / \mathrm{VEL}+\mathrm{Rbv}$ or SOF + DCV + Rbv for 24 weeks are recommended.

- Retreatment of genotype-3 infected patients who previously failed a recommended 24-weeks SOF $+\mathrm{DCV}+\mathrm{Rbv}$ regimen with Y93H RAV should not be considered for retreatment with $\mathrm{SOF}+\mathrm{VEL}+\mathrm{Rbv}$ for 24 weeks, but rather wait for new combination, whether retreatment can be delayed.

\section{Retreatment of patients failing to achieve SVR with DAA-based regimens (DAA-failures)}

Until the end of February 2017, 68,270 patients have already been treated with IFN-free DAA-combinations in Italy. According to current literature, suggesting an average of $2-3 \%$ virologic failures to current DAA regimens, approximately 2000-4000 DAA-failure patients are waiting for efficient retreatment options.

Three types of virologic failures exist, most common being relapse, that is post treatment viremia reappearance following HCV RNA undetectability at the end of therapy. Non response, defined as HCV-RNA $>1000 \mathrm{IU} / \mathrm{mL}$ at week 4 of treatment, and breakthrough defined as on-treatment detectable viremia in two controls after HCV RNA undetectability. Non response and breakthrough are rarely observed with DAA treatment, and their occurrence should raise questions on patient's adherence and/or on wrong HCVgenotype assignment.

DAA-failure can result from multiple factors, which need to be carefully evaluated when considering possible reasons for not achieving the SVR on a case-by-case basis. Indeed, treatment outcome can be affected by patient-related factors such as compliance and unknown drug-to-drug interactions (DDI) potentially reducing DAA efficacy (including over-the-counter, recreational drugs and herbal supplements). However, DAA-failure is often the result of a suboptimal treatment regimen, defined by (co)presence of multiple unfavorable factors. These include suboptimal DAA combinations use (low potency or low barrier to resistance), too short treatment duration, use of Rbv-free regimens whether treatment schedule could have required Rbv addition, previous treatment experience, baseline HCV RNA viral load and baseline RAVs.

All these baseline factors need to be retrospectively evaluated when considering reasons for treatment failure, (see next paragraph).

According to recent data, up to $4 \%$ of DAA-failures are caused by wrong baseline HCV genotype determination, especially when HCV genotype was assessed at the beginning of patient's clinical history using old-generation tests that carried increased risk of genotype/subtype misclassification [41]. Viral sequencing is thus recommended at treatment failure in order to confirm HCV genotype, especially when breakthourgh or non-responses to DAA treatment are observed. In addition, due to frequent development of RAVs at failure and their critical role in retreatment optimization, resistance testing in all three resistance-relevant NS3, NS5A, NS5B regions has always to be performed.

\subsection{RAV at virological failure and retreatment options}

Failure to DAA-based regimens invariably results in RAVs selection, as drug pressure modifies viral population, that is progressively enriched in resistant strains. At virologic failure, all patients carry RAVs to at least one DAA they have been exposed to: RAVs analysis of 261 patients failing a DAA-based regimens in a multicentre Italian study found 72\% NS3 and 93\% NS5A-RAVs prevalence in patients failing a NS3 and NS5A-containing regimen, respectively, while only $19.7 \%-31 \%$ of NS5B-RAV were detected after NS5B-containing regimens [42]. These figures are consistent with different replicative fitness exhibited by RAV types, and result in heterogeneous clinical relevance of RAVs when considering retreatment.

Indeed, aminoacidic substitutions in NS5B region result in viral strains with extremely low replicative fitness, due to the high conservation of RNA polymerase catalytic site. As a consequence, retreatment of SOF-failed patients with SOF-containing regimens yields optimal efficacy [43].

Also NS3 RAVs display lower replicative fitness compared to wild-type virus, so that resistant strains are progressively replaced by wild-type ones following the end of treatment. It has been estimated that approximately 10-12 months after PI-discontinuation, the entire viral population would have been reverted to wild-type, although outcome of NS3-based retreatment in terms of possible NS3-RAV re-emergence has not yet been defined [44].

According to EASL Guidelines, the optimal retreatment regimen for patients failing a first DAA course should contain one DAA with high barrier to resistance (i.e. NS5B nucleotide inhibitors) plus 1-3 other DAAs, with no cross-resistance with the DAAs already administered. Rbv should be added in all retreatments. A 12-week course is recommended in patients with mild fibrosis (F0-F2), where patients with advanced fibrosis (F3/4) should be retreated for 24 weeks [16]. As a consequence, NS3-RAV carriers are "easy to retreat" patients, as they can efficiently be retreated with currently available SOF plus NS5A combinations [45].

On the other hand, NS5A RAV displays the same replicative fitness as wild-type virus and persist long-term, remaining detectable more than two year after treatment failure. NS5A inhibitors have similar resistance profiles, with consequent risk of cross-resistance after failure [46-48]. According to the principle of DAA class switch, these patients could be retreated only with a NS3-containing regimens, such as SOF+SMV+Rbv. However, this regimen is not recommended in decompensated cirrhosis and could result in suboptimal antiviral efficacy in genotype 1a patients due to low SMV resistance barrier.

The most difficult therapeutic scenario is in the management of patients harboring RAVs to multiple DAA classes, for example NS5A plus NS3: these patients could be retreated only with multiple DAA combinations targeting all replication steps, such as a NS5B nucleotide polymerase inhibitor \pm NS5B non-nucleotide + NS5A + NS3 inhibitor. For example, retreatment strategies for genotype 1 patients could include SOF + GZR/EBR, $\mathrm{SOF}+3 \mathrm{D}$ combination, SOF + SMV + DCV, however these combinations are out of reimbursability policies in many countries. "Unconventional" approaches out of drug labels/market reimbursement need cooperation from drug companies and refining of national reimbursement rules to be translated in clinical practice. According to EASL guidelines, retreatment of genotype 3 patients failing SOF + DCV combination could be based on SOF/VEL + Rbv for 24 weeks, however in presence of Y93H RAV at failure these patients should not be considered for retreatment at the present time, but rather wait for future options.

Next-generation DAA combination could solve the unmet need for efficient retreatment options: indeed in the POLARIS-1 trial, SOF/VEL/VOX combination achieved 96\% SVR rates in $263 \mathrm{HCV}$ genotype 1-6 NS5A-experienced patients, and SVR rates were not affected by baseline presence of multiple RAVs (NS5A and NS3) [40]. Also the MK3 combination demonstrated optimal efficacy in genotype 1 patients with previous DAA failure. In the C-SURGE study, comparing MK3 + Rbv for 16 weeks vs MK3 for 24 weeks in 94 genotype 1 patients failing SOF/LDV or GRZ/EBR, SVR rates were above $98 \%$ independently from baseline RAV profile and treatment schedule administered [15]. Finally, the MAGELLAN-1 study, that investigated the use of GLE/PIB \pm Rbv in $50 \mathrm{DAA}$-experienced geno- 
type 1 patients, achieved 100\% SVR independently from baseline NS5A or NS3 RAVs [49].

As a consequence, if not clinically urgent, retreatment of patients with multiple RAVs to NS5A and NS3 could be deferred, waiting for upcoming new treatment options.

\subsection{Statements}

- Resistance testing after treatment failure in all 3 genes (NS3, NS5A, NS5B [for the two different classes of nucleoside and nonnucleoside inhibitors] independently from the failure regimen) is mandatory in order to optimize retreatment strategy. ${ }^{1}$

- HCV sequencing can be based on Sanger population method or by ultra-deep sequencing using a cut-off of $15 \%$ prevalence. It can confirm the previous genotype and subtype assignment, but can also attribute a different HCV-genotype highlighting cases of previous wrong genotype assignation by commercial assays or occurrence of potential re-infection instead of virological relapse, avoiding then inappropriate retreatment regimens.

- Resistance testing should be performed as soon as possible after documentation of failure, provided that HCV RNA has reached a value of at least $1000 \mathrm{IU} / \mathrm{mL}$, according to the threshold limits for virology laboratories for HCV sequencing.

- Clinical and virological information required for resistance test performance and interpretation should be provided contextually with blood samples.

- According to resistance results, current re-treatment strategies for patients failing a first course with DAAs should include at least 2 active drug classes, with a preferential use of one drug with high genetic barrier to resistance, and with extended treatment durations and addition of Rbv, otherwise waiting for better future options is recommended.

- If a deferred treatment has been considered, and in case of presence of RAV at failure, in order to assist the therapeutic choice when starting a re-treatment, HCV sequencing should be repeated (only in the gene with previous RAV) and should be based on deep sequencing.

- Retreatment of genotype 3 patients who previously failed a recommended 24-weeks SOF + DCV + Rbv regimen with Y93H RAV should not be considered for retreatment with SOF/VEL + Rbv for 24 weeks, but rather wait for new combinations, if retreatment can be delayed.

\section{Baseline features influencing HCV treatment choice}

When evaluating treatment options, choice of the best treatment regimen and schedule at the individual level should consider many baseline factors, such as HCV genotype, viral load, liver fibrosis stage and previous treatment experience, baseline RAV, DDI and patient comorbidities. Careful evaluation of these baseline features can help to optimize treatment strategy and maximize its efficacy.

\subsection{HCV genotype}

Some DAA-based regimens are still genotype/subtype dependent, so that a precise assessment of HCV genotype and subtype is mandatory in order to avoid mistreating during the first DAA course. In many patients, HCV genotype and subtype have been determined at the beginning of clinical history, often using old-

\footnotetext{
1 Note: It would be desirable to preserve a sample before starting treatment with DAA, because in case of failure and presence of RAV, the study of the baseline sample may help to distinguish if the resistance occurred on treatment failure or if it was already present as natural resistance before treatment. This information may help to set to the best the next regimen.
}

generation tests, i.e. the commercial assay INNO-LIPA version 1.0; these tests carried an increased risk of misclassifying genotype 1a subtype, that nowadays requires addition of Rbv or treatment extension in many DAA regimens. The currently available second-generation commercial assays for HCV genotype show good concordance (around 90\%) with genotype assessment performed by HCV sequencing in the NS5B, or NS3, or NS5A region [50], and are now considered the standard of care for HCV genotyping, so that HCV sequencing is not routinely recommended. However, HCV sequencing can be useful in those rare cases where genotype cannot be determined by the commercial assays (such as cases of mixed infection, or indeterminate, or genotype 1 without subtype information).

\subsection{HCV RNA viral load}

When choosing treatment regimen, viral load should be considered in some selected cases, where HCV RNA levels drive treatment duration or need for Rbv addition. This is the case for SOF/LDV treatment in naive genotype 1 patients without cirrhosis, who can be treated with a shortened 8-week course of SOF/LDV if HCV RNA is $<6,000,000 \mathrm{IU} / \mathrm{mL}$, according to a sub-analysis of ION-3 trial [51]. In genotype 4 Peg-IFN-experienced patients and in all genotype $1 \mathrm{a}$ patients with HCV RNA $>800,000 \mathrm{IU} / \mathrm{mL}$, treatment duration with GZR/EBR combination should be extended from 12 to 16 weeks with Rbv addition, as a pooled analysis of Phase II and Phase III GZR/EBR trials showed suboptimal rates in patients with HCV RNA $>800,000 \mathrm{IU} / \mathrm{mL}$ and baseline RAV to EBR receiving GZR/EBR for 12 weeks without Rbv [52,53]. These data generated the drug label recommendations, where baseline RAV sequencing, if available or baseline HCV RNA lead choice of treatment duration and need for Rbv use.

\subsection{Liver fibrosis stage}

Liver disease assessment is mandatory in order to optimize treatment strategy: intensified treatment courses (extension to 24 weeks and/or Rbv addition) are required with most DAA combinations in presence of cirrhosis. Moreover, NS3-containing regimens are not recommended in decompensated Child B or $\mathrm{C}$ patients (3D/2D, SMV and GRZ/EBR), as liver impairment results in increased exposure to NS3 inhibitor concentrations and, consequently, enhanced risk of adverse events.

Liver staging can be assessed by non-invasive methods (like Transient Elastography) and, in cirrhotic patients, detailed medical history concerning previous episodes of liver decompensation should be recorded, together with baseline Child Pugh score assessment, in order to define the potential risk of decompensation and liver toxicity.

\subsection{Previous treatment experience}

EASL Guidelines recommend different treatment regimens in naive vs Peg-IFN experienced patients with many DAA combinations, suggesting Rbv addition or treatment extension [16]. This is the case for SOF/LDV combination, where genotype 1a, 4-6 experienced patients should be treated with Rbv addition. The same concept is true for genotype 3 patients receiving SOF/VEL or $\mathrm{SOF}+\mathrm{DCV}$ treatment, where experienced patients benefit from Rbv use, especially in presence of baseline Y93H RAV. When considering GZR/EBR combination, treatment extension to 16 weeks and Rbv addition is recommended in genotype 1 a treatment experienced patients with baseline viral load $>800,000 \mathrm{IU} / \mathrm{mL}$. 


\subsection{Baseline RAV}

Due to the lack of proof-reading activity of the HCV RNA polymerase and the high HCV replication rate, RAVs naturally occur and preexist DAA exposure, being detected with different prevalence according to the sequencing technique (population-based sequencing vs deep sequencing). Potential role of baseline RAV in determining treatment outcome has been widely discussed: a sub-analysis of the SOF/LDV phase II and III clinical trials showed that baseline NS5A LDV-specific RAV negatively affected treatment outcome in some patient subsets, such as genotype 1a treatmentexperienced patients. Baseline LDV-RAV were detected in $8-16 \%$ of patients using a $15 \%$ viral sequencing cut off [54]. On the other hand, another study demonstrated that baseline RAV had only minimal effect in genotype 1 a patients receiving SOF/LDV combination, and RAV could be overcome by treatment extension or Rbv addition [55]. EASL guidelines recommend Rbv addition in genotype 1a experienced patients with SOF/LDV or SOF + DCV regimens in presence of baseline RAV that confer high-level resistance to NS5A inhibitors, however RAS testing is not mandatory if not available.

Another treatment choice influenced by baseline RAV is treatment duration of GZR/EBR combination therapy, where SVR rates in patients treated for 12 weeks without Rbv dropped to $52 \%$ and $29 \%$ in naive and experienced genotype 1 a patients, respectively. These data led drug label to recommend RAV sequencing at baseline in order to extend treatment duration to 16 weeks and add Rbv in patients harboring RAV to EBR. If RAV testing is not available, a baseline viral load $>800,000 \mathrm{IU} / \mathrm{mL}$ is the surrogate cut-off to drive treatment intensification [16].

Finally, addition of Rbv has been recommended in treatment experienced genotype 3 patients receiving SOF +DCV or SOF/VEL combination in presence of baseline RAV Y93H, resulting in suboptimal SVR rates in ALLY-3 and ASTRAL-3 trials [31,36].

\subsection{Drug-drug interactions (DDIs)}

Careful evaluation of patient's co-medications (including overthe-counter, herbals and supplements) is mandatory when choosing the appropriate treatment regimen, in order to avoid significant DDIs resulting in reduced treatment efficacy or risk for significant side effects. One of the most relevant DDIs is between SOF and amiodarone, whose co-administration is contraindicated due to risk of severe bradycardia. While the pathogenic mechanism has not been completely elucidated yet, this contraindication has been extended to all SOF-containing regimens and, due to amiodarone accumulation, a wash-out period of minimum three months has been recommended prior to SOF-based treatments. Other significant DDIs resulting in contraindicated co-administration include statins with 3D regimen (rosuvastatin with SOF/LDV), and cyclosporine with GRZ/EBR, due to toxicity risk resulting from increased GZR concentrations. Finally, many anticonvulsant drugs like phenobarbital and carbamazepine cannot be co-administered with many DAAs because they decrease antiviral treatment efficacy.

Most common drug classes involved in DDIs with DAAbased regimens are cardiovascular (antihypertensive, antiarrhythmic agents), antipsychotic and immunosuppressive drugs (tacrolimus, cyclosporine, mycophenolate), some anti-platelets and anti-fibrinolytic agents. First-generation DAAs, especially NS3 inhibitors, are characterized by more DDIs compared to NS5A inhibitors, due to the metabolic pathway involving cytocrome CYP3A4, which is common to many other drugs. While in some cases medication change is required during antiviral treatment, more often only a careful monitoring for side effects is needed. Second-generation drugs represent a significant improvement in DDI profile, resulting in easier management of patient's comedications.

\subsection{Co-morbidities}

Patient's comorbidities (including HIV and HBV co-infection, cardiovascular and renal diseases, cryoglobulinemia and haematologic diseases) play an important role in treatment eligibility and DAA-combination decision. Some comorbidities confer urgency criteria for antiviral treatment independently from liver disease stage, i.e. EHMs such as HCV-related cryoglobulinemia and non-Hodgkin lymphoma. While HIV-infected patients are now considered as mono-infected patients according to treatment efficacy, choice of treatment course is strongly influenced by DDIs with antiretroviral therapy. Patients with concurrent HBV infection require careful monitoring and/or HBV treatment in order to prevent HBV reactivation during anti-HCV therapy.

Most relevant co-morbidities affecting treatment decision are cardiovascular and renal diseases. In the first case, DDIs with cardiovascular agents have to be carefully evaluated and can result in contraindicated antiviral treatment (i.e. amiodarone) or need for medication changes. In addition, patients with cardiovascular diseases should be treated, whenever possible, with Rbv-free regimens, due to the potential risk of side effects resulting from anemia development.

DAA-based treatment options for HCV patients with chronic kidney disease (CKD) are still restricted, as administration of SOFbased regimens is not recommended in patients with a glomerular filtration rate (eGFR) below $30 \mathrm{~mL} / \mathrm{min}$. This recommendation results from pharmacokinetic data showing accumulation of SOF metabolite GS-331007 in patients with impaired renal function [56]. While clinical relevance of the increased exposure to GS331007 is still debated, many real-life experiences have reported SOF-based therapy of $\mathrm{HCV}$ patients with GFR $<30 \mathrm{~mL} / \mathrm{min}$ with optimal efficacy, using different treatment schedules (SOF full-dose $400 \mathrm{mg} /$ day, $200 \mathrm{mg} /$ day or $400 \mathrm{mg} /$ three times a week) [57]. However, SOF-based treatment of advanced CKD patients can result in increased side effects, mainly anemia, and some patients showed also renal function deterioration on-therapy, as reported in the large TARGET study [58]. The only approved regimens for CKD stage 4-5 (GFR $<30 \mathrm{~mL} / \mathrm{min}$ ) patients are 3D/2D regimen and GZR/EBR combination, which are effective only in genotype 1 and 4 patients and are not recommended in decompensated cirrhosis. The RUBY1 study evaluated the efficacy and safety of $3 \mathrm{D} \pm \mathrm{Rbv}$ for 12 weeks in 20 genotype 1 CKD stage $4-5$ patients: 18/20 (90\%) patients achieved SVR and anemia was the only side effect in genotype 1a patients, where Rbv administration was prescribed [59]. The following RUBY-2 study compared 3D regimen for 12 weeks with or without Rbv in genotype 1a CKD patients, showing no significant difference in treatment efficacy according to Rbv use [60]. Concerning GZR/EBR combination, the phase 3 C-SURFER study showed 99\% SVR rates in 224 genotype 1 patients treated for 12 weeks, with optimal safety profile as a consequence of the Rbv-free regimen [61]. In addition, GZR/EBR displays reduced DDIs compared to $3 \mathrm{D} / 2 \mathrm{D}$ regimens, due to presence of second-generation DAA compounds. Genotype 2 or 3 patients with advanced CKD have now limited treatment options except from off-label use of SOF-based regimens, however the pan-genotypic second generation NS3 + NS5A GLE/PIB combination, which is not renally excreted, is expected to provide efficient treatment options: indeed the EXPEDITION-4 trial reported 98\% SVR in 104 genotype 1-6 CKD patients [13].

\subsection{Statements}

- Treatment choice should consider baseline factors including HCV genotype, viral load (in selected cases), liver fibrosis stage and previous treatment experience, drug-to-drug interactions and patient co-morbidities. 
Table 1

Baseline demographic and virological characteristics of the included patients.

\begin{tabular}{ll}
\hline Characteristics & $\mathrm{N}=23,384$ \\
\hline Genotype, $\mathrm{n}(\%)$ & \\
1a & $3060(13 \%)$ \\
1b & $13,230(56 \%)$ \\
2 & $3256(14 \%)$ \\
3 & $2497(11 \%)$ \\
4 & $1341(6 \%)$ \\
Age, years ${ }^{\mathrm{a}}$ & $59(21-90)$ \\
Fibrosis stage, n (\%) & \\
F3 & $6448(28 \%)$ \\
F4 & $15,388(66 \%)$ \\
Liver stiffness, $\mathrm{kPa}^{\mathrm{a}}$ & $18.3(2-75)$ \\
Platelets $\mathrm{x} 10^{3} / \mathrm{mm}^{3 \mathrm{a}}$ & $141(12-990)$ \\
Albumin, $\mathrm{g} / \mathrm{dL}^{\mathrm{a}}$ & $3.9(1.9-5.6)$ \\
Bilirubin, $\mathrm{mg} / \mathrm{dL}^{\mathrm{a}}$ & $0.98(0.1-19)$ \\
On waiting list for LT, n (\%) & $103(0.4 \%)$ \\
HIV positive, $\mathrm{n}(\%)$ & $1531(6.5 \%)$ \\
\hline
\end{tabular}

a Median (range).

- Accurate assessment of HCV genotype and subtype in genotype 1 by a II generation commercial assay is mandatory before starting a DAA-based treatment. HCV sequencing is recommended in all rare cases where genotype or subtype cannot be determined by the commercial assays.

- Baseline resistance testing for RAV before first course with DAAs has limited clinical utility, unless if recommended by specific drug labels.

\section{Real life experience with DAAs in Italy}

In order to assess the demographic, clinical and virological features of patients treated with DAAs and the overall efficacy of different anti-HCV treatments in Italian real-life practice, we retrospectively collected the data of four Regional Hepatitis C Registry (Campania, Lombardia, Sicilia and Veneto) including all CHC infected patients over 18 years old who consecutively received DAA-based treatment. Overall, between January 2015 and the end of 2016, 23,384 patients were treated in 101 treatment Centres based on the AIFA criteria (Table 1). The treatment' regimens were chosen by physician according to European (EASL) and Italian (AISF) liver societies guidelines. Stage of liver disease was assessed on clinical ground, by liver biopsy or liver stiffness measurements (LSM) considering as advanced liver fibrosis LSM $\geq 10 \mathrm{kPa}$. Analysis of treatment outcome is available in 12,595 (54\%) patients who completed treatment or follow up (Table 2). Overall SVR rate was
95.6\% (12,038/12,595): 96\% in genotype 1a (1447/1506); $96.6 \%$ in genotype $1 \mathrm{~b}$ (7229/7482); $95 \%$ in genotype 2 (1558/1642), $91.2 \%$ in genotype $3(1184 / 1298)$ and $93 \%$ in genotype $4(620 / 667)$. The cumulative rate of HCC in cirrhotic patients with and without SVR was, respectively: $2.9 \%$ vs $15.2 \%$.

The strength of combining the data of the four largest Italian registers on DAAs is based on the huge number of patients and the high number of prescribing centers with different volumes of treated patients to fully justify the definition of "real clinical practice". Despite our collection may lack some important data at this time, it confirms that in clinical practice the effectiveness of DAAs regimens are equal to those reported in registration trials who have allowed the approval of some treatment regimens with limited data in particular subgroups of patients.

\section{Conclusion}

The availability of highly effective and well-tolerated DAAs has changed the approach to treating $\mathrm{CHC}$ patients. Data from real life reflect and in some cases exceed those reported in the pivotal trials and today we are able to cure infection and halt liver disease progression reducing both liver-related and overall mortality. Moreover data accumulated in the last few years from real life were useful to improve patient's treatment both in term of efficacy and safety.

Most of the clinical needs on management of $\mathrm{CHC}$ patients are resolved compared to last year [62]. With new pan-genotypic DAA combinations, effective against all viral genotypes and all stage of liver disease however we can further improve the management of our patients with prior treatment failure or with severe renal impairment, moreover with treatments of shorter duration durations. In the next future we might finally have the potential to treat the infection and not just the disease, with the aim to eradicate HCV worldwide.

\section{Conflict of interest}

Viganò M: Consultant/advisor/sponsored lectures for BMS, Gilead Sciences, Roche.

Perno CF: Research grants, lecturing fees, advisory boards, scientific consultancies for Abbvie, Gilead Sciences, BMS, Janssen Cilag, VIIV, Roche, Abbott Diagnostics.

Craxì A: Research grants, lecturing fess, advisory boards, scientific consultancy for Novartis, Abbvie, Gilead Sciences, BMS, Achillion, Tibotech, Janssen Cilag, Abbott Diagnostics.

Table 2

Effectiveness of the different treatment regimens according to HCV genotype.

\begin{tabular}{|c|c|c|c|c|c|}
\hline \multirow[t]{2}{*}{ Regimens } & \multicolumn{5}{|c|}{ Number of patients with SVR/number of patients with available treatment outcome (\%) } \\
\hline & Genotype 1a & Genotype 1b & Genotype 2 & Genotype 3 & Genotype 4 \\
\hline $\mathrm{SOF} / \mathrm{LED} \pm \mathrm{Rbv} 12$ weeks & $412 / 432(95.3 \%)$ & $1173 / 1209(97 \%)$ & - & - & $118 / 130(90.8 \%)$ \\
\hline $\mathrm{SOF} / \mathrm{LED} \pm \mathrm{Rbv} 24$ weeks & $324 / 331(97.9 \%)$ & $1515 / 1547(98 \%)$ & - & & $109 / 116(93.9 \%)$ \\
\hline SOF + SIM 12 weeks & $58 / 60(96.7 \%)$ & $260 / 279(93.2 \%)$ & - & - & - \\
\hline $\mathrm{SOF}+\mathrm{SIM}+\mathrm{Rbv} 12$ weeks & $258 / 280(92.1 \%)$ & $1425 / 1519(93.8 \%)$ & - & - & $146 / 162(90.1 \%)$ \\
\hline DCV + SOF 12 weeks & - & - & $199 / 202(98.5 \%)$ & $45 / 50(90 \%)$ & - \\
\hline DCV + SOF 24 weeks & $26 / 28(92.8 \%)$ & $123 / 126(97.6 \%)$ & - & $122 / 130(93.8 \%)$ & $6 / 6(100 \%)$ \\
\hline $\mathrm{DCV}+\mathrm{SOF}+\mathrm{Rbv} 12$ weeks & $28 / 28(100 \%)$ & $106 / 106(100 \%)$ & - & $63 / 65(96.9 \%)$ & $12 / 12(100 \%)$ \\
\hline $\mathrm{DCV}+\mathrm{SOF}+\mathrm{Rbv} 24$ weeks & $74 / 74(100 \%)$ & $228 / 230(99.1 \%)$ & - & $656 / 671(97.8 \%)$ & $16 / 16(100 \%)$ \\
\hline 3D + Rbv 12 weeks & $101 / 104(97.1 \%)$ & $991 / 1011(98 \%)$ & - & & - \\
\hline 3D + Rbv 24 weeks & $166 / 169(98.2 \%)$ & $1360 / 1383(98.3 \%)$ & - & & - \\
\hline $2 \mathrm{D}+\mathrm{Rbv} 12$ weeks & - & - & - & - & $80 / 82(97.5 \%)$ \\
\hline $2 \mathrm{D}+\mathrm{Rbv} 24$ weeks & - & - & - & - & $122 / 124(98.4 \%)$ \\
\hline $\mathrm{SOF}+\mathrm{Rbv} 12$ weeks & - & - & $601 / 633$ (94.9\%) & - & - \\
\hline SOF + Rbv 24 weeks & - & $48 / 72(66.7 \%)$ & $758 / 807(93.9 \%)$ & $264 / 345(76.5 \%)$ & $11 / 19(57.9 \%)$ \\
\hline SOF/PegIFN/Rbv 12 weeks & - & - & & $34 / 37(91.9 \%)$ & - \\
\hline
\end{tabular}

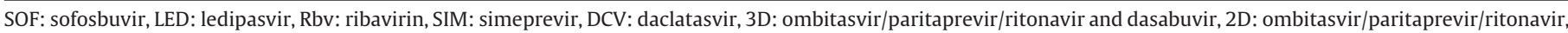
PegIFN: pegylated interferon. 
Aghemo A: Advisory Boards: BMS, MSD, Gilead Sciences, Janssen, AbbVie; Speaker: BMS, MSD, Gilead Sciences, Janssen, AbbVie: Research Grant: Gilead Sciences.

Alberti A: Advisor board e speaker fee: Gilead Sciences, Abbivie, BMS MSD, Janssen; Research founding: Gilead Sciences, Janssen, Abvvie, MSD.

Andreone P: Research grants from Gilead Sciences, MSD and Roche; advisor for Janssen, MSD, Roche, AbbVie, BMS, Gilead Sciences; consultant for BMS, MSD, AbbVie.

Andreoni M: Attending symposia, speaking, organizing educational activities, consultancy or advisory board membership, or grant research support from MS Gilead Sciences, BMS, Janssen, Viiv Healthcare, MSD, AbbVie, Astra Zeneca, Boheringer Ingelheim, Pfizer.

Bonora S: Recipient of travel grants, advisory board fees or speaker fees from MSD, Viiv, Janssen, BMS, Abbvie and Gilead Sciences.

Brunetto MR: Speakers' bureau: BMS, Gilead Sciences, Janssen, Roche; advisory board: Gilead Sciences, Janssen, Abbvie, MSD.

Bruno R: Advisory board and speaker Abbvie, BMS, Gilead, Janssen, MSD.

Bruno S: Advisory board MSD, AbbVie, speaker bureau MSD, speaker AbbVie, BMS, Gilead Sciences, Janssen, MSD.

Calvaruso V: Travel grant, speaking, teaching and participation to advisory board for: AbbVie, BMS, Gilead Sciences and Intercept. Grant and research support: MSD.

Caporaso N: Speaking and teaching Abbvie, BMS, Gilead Science, Janssen, MSD. Advisory committees of Abbvie, BMS, Gilead Science, Janssen, MSD.

Ceccherini-Silberstein F: Attending symposia, speaking, organizing educational activities, consultancy or advisory board membership, or grant research support from AbbVie, Abbott Molecular, BMS, Gilead Sciences, Janssen-Cilag, MSD, Roche Diagnostics and ViiV Healthcare.

Cento V: Advisory boards and/or lecturer for Abbvie, BMS, MSD, Janssen-Cilag.

Ciancio A: Speaker bureau for MSD; BMS, Gilead Sciences, Abbvie.

Colombatto P: Advisory board AbbVie; speaker bureau: Janssen e BMS.

Degasperi E: None.

Di Marco V: Speaking and advisor for: AbbVie, BMS, Gilead, MSD, Roche. Grant and research support: Abbvie, Bayer, BMS, Gilead, MSD, Roche.

Di Perri G: Speaker and advisor for: Abbvie, BMS, MSD, Gilead Sciences, Janssen, ViiV, Pfizer, Novartis, Aventis.

D'offizi G: Speaking, teaching and participation to advisory board for: Gilead, BMS, MSD.

Fagiuoli S: Speaker bureau: Gilead Sciences, BMS, MSD, Abbvie, Janssen, Novartis, Kedrion, Biotest, Bayer.

Ferrari C: Research grants from Gilead Sciences, Roche, Janssen; served as advisor/consultant for Gilead Sciences, MSD, Abbvie, BMS and Arrowhead.

Gaeta GB: Speaker or Advisor for: MSD, Gilead Sciences, BMS, Abbvie, Janssen, Roche.

Pellicelli A: None.

Petta S: Consultant/Advisor/Sponsored lectures for Abbvie, BMS, Gilead Sciences, Janssen-Cilag, MSD.

Piovesan S: None.

Puoti M: Advisory boards and/or lecturer in own events and/or research grants from Abbvie, Janssen, Gilead, BMS, MSD, Roche, ViiV.

Raimondo G: Grant/research support: Gilead Sciences, MSD; Consultant/Advisor: Abbvie, BMS, Gilead Sciences, MSD, Roche; Sponsored lectures: Abbvie, Bayer, BMS, Gilead Sciences, MSD, Roche.
Russo FP: Research grant from Abbvie and BMS, lecturing fees, advisory boards for Abbvie, Gilead Sciences, Biotest, BMS, Janssen Cilag.

Taliani G: Travel grant, speaking, teaching and participation to advisory board for: AbbVie, BMS, Gilead Sciences, MSD, Janssen, Roche.

Trama U: None.

Villa E: Advisory committees or review panels: Abbvie, BMS, Gilead Sciences, GlaxoSmithKline, MSD, Roche; speaking and teaching: Abbvie, GlaxoSmithKline, Novartis.

Zignego AL: Speaker bureau for BMS, Gilead Sciences, MSD, Janssen.

\section{Appendix A}

a Division of Gastroenterology and Hepatology, Fondazione IRCCS Ca' Granda Ospedale Maggiore Policlinico, University of Milan, Milan, Italy

${ }^{\mathrm{b}}$ Department of Molecular Medicine, University of Padua, Padua, Italy

'Department of Medical and Surgical Sciences, Bologna University, Bologna, Italy

${ }^{\mathrm{d}}$ Department of Infectious Diseases, University of Rome Tor Vergata, Rome, Italy

eUnit of Infectious Diseases, Department of Medical Sciences, University of Turin, Turin, Italy

${ }^{\mathrm{f}}$ Hepatology Unit, University Hospital of Pisa, Pisa, Italy

gUnit of Infectious and Tropical Diseases, University of Pavia, Pavia, Italy

h Department of Internal Medicine, Humanitas University Medicine, Rozzano, Italy

i Department of Gastroenterology, DiBiMIS, University of Palermo, Palermo, Italy

${ }^{j}$ Department of Clinical Medicine and Surgery, Gastroenterology Unit, University of Naples F̈ederico II; Naples, Italy

kVirology Unit, Department of Experimental Medicine and Surgery, "Tor Vergata" University of Rome, Rome, Italy

lDepartment of Gastroenterology and Hepatology, Città della Salute e della Scienza Hospital and Department of Medical Sciences, University of Turin, Turin, Italy

mUnit of Infectious Diseases and Hepatology, INMI “Lazzaro Spallanzani" Rome, Italy

${ }^{\mathrm{n}}$ Department of Gastroenterology, Hepatology and Liver Transplantation, Papa Giovanni XXIII Hospital, Bergamo, Italy

'Unit of Infectious Diseases and Hepatology, AOU di Parma, Parma, Italy

PSection of Infectious Diseases, Department of Internal Medicine, Second University of Naples, Naples, Italy

qLiver Unit, San Camillo Forlanini Hospital, Rome, Italy

'Division of Infectious Diseases, AO Ospedale Niguarda Ca' Granda, Milan, Italy

${ }^{s}$ Division of Clinical and Molecular Hepatology, Department of Internal Medicine, University Hospital of Messina, Messina, Italy

tSection of Gastroenterology/Multivisceral Transplant Unit, Department of Surgery, Oncology and Gastroenterology, Padua University Hospital, Padua, Italy

unfectious and Tropical Diseases Unit, Department of Clinical Medicine, Sapienza University of Rome, Rome, Italy

vUOD 08 (Politica del Farmaco) della Regione Campania, Italy

wDivision of Gastroenterology, AOU Policlinico di Modena, University of Modena and Reggio Emilia, Modena, Italy

${ }^{x}$ Center for Systemic Manifestations of Hepatitis Viruses (MASVE), Department of Experimental and Clinical Medicine, University of Florence, Florence, Italy 


\section{References}

[1] Webster DP, Klenerman P, Dusheiko GM. Hepatitis C. The Lancet 2015;385:1124-35

[2] GBD 2013 Mortality and Causes of Death Collaborators. Global, regional, and national age-sex specific all-cause and cause-specific mortality for 240 causes of death, 1990-2013: a systematic analysis for the Global Burden of Disease Study 2013. The Lancet 2015;385:117-71.

[3] Alter MJ. Epidemiology of hepatitis C virus infection. World Journal of Gastroenterology 2007;13:2436-41.

[4] Messina JP, Humphreys I, Flaxman A, et al. Global distribution and prevalence of hepatitis C virus genotypes. Hepatology 2015;61:77-87.

[5] van der Meer AJ, Veldt BJ, Feld JJ, et al. Association between sustained virological response and all-cause mortality among patients with chronic hepatitis $\mathrm{C}$ and advanced hepatic fibrosis. JAMA 2012;308:2584-93.

[6] Deterding K, Hoener zu Siederdissen C, Port K, et al. Improvement of liver function parameters in advanced HCV associated liver cirrhosis by IFN-free antiviral therapies. Alimentary Pharmacology Therapeutics 2015;42:889-901.

[7] Jacobson IM, Asselah T, Nahass R, et al. A randomized phase 3 trial of Sofosbuvir/Velpatasvir/Voxilaprevir for 8 weeks compared to Sofosbuvir/Velpatasvir for 12 weeks in DAA-naïve genotype 1-6 HCV infected patients: the POLARIS-2 study. Hepatology 2016;64(Suppl. S1) [Abstract LB12].

[8] Zeuzem S, Flamm SL, Tong M, et al. A randomized, controlled, phase 3 trial of Sofosbuvir/Velpatasvir/Voxilaprevir or Sofosbuvir/Velpatasvir for 12 weeks in direct-acting antiviral-experienced patients with genotype 1-6 HCV infection: the POLARIS-4 study. Hepatology 2016;64(Suppl. S1) [Abstract 109].

[9] Zeuzem S, Feld J, Wang S, et al. ENDURANCE-1: a phase 3 evaluation of the efficacy and safety of 8-versus 12-week treatment with Glecaprevir/Pibrentasvir (formerly ABT-493/ABT-530) in HCV genotype 1 infected patients with or without HIV-1 co-infection and without cirrhosis. Hepatology 2016;64(Suppl. S1) [Abstract 253].

[10] Asselah T, Hezode, Zadeikis N, et al. ENDURANCE-4: efficacy and safety of Glecaprevir/Pibrentasvir (Formerly ABT-493/ABT-530) treatment in patients with chronic HCV genotype 4, 5, or 6 infection. Hepatology 2016;64(Suppl. S1) [Abstract 114].

[11] Kowdley KV, Colombo M, Zadeikis N, et al. ENDURANCE-2: safety and efficacy of Glecaprevir/Pibrentasvir in hepatitis $C$ virus genotype 2-infected patients without cirrhosis: a randomized, double-blind, placebo-controlled study. Hepatology 2016;(Suppl. S1):64 [Abstract 73].

[12] Wyles D, Poordad F, Wang S, et al. SURVEYOR-II, part 3: efficacy and safety of Glecaprevir/Pibrentasvir (ABT-493/ABT-530) in patients with hepatitis C virus genotype 3 infection with prior treatment experience and/or cirrhosis. Hepatology 2016;64(Suppl. S1) [Abstract 113].

[13] Gane E, Lawitz E, Pugatch D, et al. EXPEDITION-4: efficacy and safety of Glecaprevir/Pibrentasvir (ABT-493/ABT-530) in patients with renal impairment and chronic hepatitis $C$ virus genotype 1-6 infection. Hepatology 2016;64(Suppl. S1) [Abstract LB11].

[14] Lawitz E, Yoshida EM, Buti M, et al. Safety and efficacy of the fixed-dose combination regimen of MK-3682/Grazoprevir/MK-8408 (Ruzasvir) with or without ribavirin in non-cirrhotic or cirrhotic patients with chronic HCV GT1, 2 or 3 infection (Part B of C-CREST-1 \& -2). Hepatology 2016;64(Suppl. S1) [Abstract 110].

[15] Wyles D, Wedemeyer H, Reddy KR, et al. Safety and efficacy of the fixed-dose combination regimen of MK-3682/Grazoprevir/MK-8408 (Ruzasvir) in cirrhotic or non-cirrhotic patients with chronic HCV GT1 infection who previously failed a direct-acting antiviral regimen (C-SURGE). Hepatology 2016;64(Suppl. S1) [Abstract 193].

[16] EASL. Recommendations on treatment of hepatitis C. Journal of Hepatology 2017;66:153-94.

[17] Sulkowski MS, Gardiner DF, Rodriguez-Torres M, et al. Daclatasvir plus sofosbuvir for previously treated or untreated chronic HCV infection. New England Journal of Medicine 2014:370:211-21.

[18] Poordad F, Schiff ER, Vierling JM, et al. Daclatasvir with sofosbuvir and ribavirin for hepatitis C virus infection with advanced cirrhosis or post-liver transplantation recurrence. Hepatology 2016;63:1493-505.

[19] Zhou N, Han Z, Hartman-Neumann S, et al. Characterization of NS5A polymorphisms and their impact on response rates in patients with HCV genotype 2 treated with daclatasvir-based regimens. Journal of Antimicrobial Chemotherapy 2016:71:3495-505

[20] Mangia A, Arleo A, Copetti M, et al. The combination of daclatasvir and sofosbuvir for curing genotype 2 patients who cannot tolerate ribavirin. Liver International 2016;36:971-6.

[21] Cacciola I, Petta S, Distefano M, et al. Efficacy of oral direct acting antivirals for treatment of advanced chronic hepatitis or compensated cirrhosis due to hepatitis C virus infection: the real-life experience of the Sicily registry. Digestive and Liver Disease 2017;49S:e6 [OC-11]

[22] Feld JJ, Jacobson IM, Hézode C, et al. Sofosbuvir and Velpatasvir for HCV genotype 1, 2, 4, 5, and 6 infection. New England Journal of Medicine 2015;373:2599-607.

[23] Foster GR, Afdhal N, Roberts SK, et al. Sofosbuvir and Velpatasvir for HCV genotype 2 and 3 infection. New England Journal of Medicine 2015;373:2608-17.

[24] Tacke F, Günther R, Buggisch P, et al. Treatment of HCV genotype 2 with sofosbuvir and ribavirin results in lower sustained virological response rates in real life than expected from clinical trials. Liver International 2017;37:205-11.
[25] Welzel TM, Nelson DR, Morelli G, et al. Effectiveness and safety of sofosbuvir plus ribavirin for the treatment of HCV genotype 2 infection: results of the real-world, clinical practice HCV-TARGET study. Gut 2016 [Epub ahead of print].

[26] Curry MP, O'Leary JG, Bzowej N, et al. Sofosbuvir and Velpatasvir for HCV in patients with decompensated cirrhosis. New England Journal of Medicine 2015;373:2618-28.

[27] Susser S, Dietz J, Barak M, et al. Prevalence and clinical importance of hepatitis C virus genotype 2 K/1 B chimeras. Journal of Hepatology 2016;64:S136.

[28] Susser S, Dietz J, Vermehren J, et al. European Ravs database: frequency and characteristics of Ravs in treatment-naïve and DAA-experienced patients. Journal of Hepatology 2016:64:S139.

[29] AASLD/IDSA HCV Guidance Panel. Hepatitis C Guidance: AASLD-IDSA recommendations for testing, managing, and treating Hepatitis C [http://www. hcvguidelines.org].

[30] Nelson DR, Cooper JN, Lalezari JP, et al. All-oral 12-week treatment with daclatasvir plus sofosbuvir in patients with hepatitis C virus genotype 3 infection: ALLY-3 phase III study. Hepatology 2015;61:1127-35.

[31] McPhee F, Hernandez D, Zhou N, et al. Next-generation sequencing analysis of NS5A and NS5 B minor resistance-associated variants in patients with HCV genotype 3 infection who failed treatment with Daclatasvir plus Sofosbuvir. Journal of Hepatology 2016;64:S614-5.

[32] Leroy V, Angus P, Bronowicki JP, et al. Daclatasvir, sofosbuvir, and ribavirin for hepatitis $C$ virus genotype 3 and advanced liver disease: a randomized phase III study (ALLY-3+). Hepatology 2016;63:1430-41.

[33] Pasulo L, Aghemo A, Quirino T, et al. Genotype 3 infection in DAA era: reports of a real life Northern Italy Network for viral hepatitis after 2 years by the start. Digestive and Liver Disease 2017;49S:e68 [F-47].

[34] Pellicelli A, Messina V, Tarquini P, et al. Daclatasvir/sofosbuvir and ribavirin $800 \mathrm{mg}$ flat dose is highly efficacy and safe in genotype 3 compensated and decompensated cirrhotic patients: the CLEO experience. Digestive and Liver Disease 2017;49S:e64 [F-39].

[35] Hézode C, Lebray P, De Ledinghen V, et al. Daclatasvir plus sofosbuvir, with or without ribavirin, for hepatitis C virus genotype 3 in a French Early Access Programme. Liver International 2017 [Epub ahead of print].

[36] Foster GR, Thompson AJ, Ruane PJ, et al. A randomized phase 3 trial of Sofosbuvir/Velpatasvir/Voxilaprevir for 8 weeks and Sofosbuvir/Velpatasvir for 12 weeks for patients with genotype 3 HCV infection and cirrhosis: the POLARIS-3 study. Hepatology 2016;64(Suppl. S1) [Abstract 258].

[37] Welzel TM, Petersen J, Herzer K, et al. Daclatasvir plus sofosbuvir, with or without ribavirin, achieved high sustained virological response rates in patients with HCV infection and advanced liver disease in a real-world cohort. Gut 2016;65:1861-70.

[38] Muir AJ, Strasser S, Wang S, et al. High Svr rates with ABT-493 + ABT-530 co-administered for 8 weeks in non-cirrhotic patients with HCV genotype 3 infection. Journal of Hepatology 2016;64:S186.

[39] Foster GR, Gane E, Asatryan A, et al. Endurance-3: a phase 3, randomized, open-label, active-controlled study to compare efficacy and safety of ABT493/ABT-530 to Sofosbuvir co-administered with Daclatasvir in adults with HCV genotype 3 infection. Journal of Hepatology 2016;64:S292.

[40] Bourlière M, Gordon SC, Ramji A, et al. Sofosbuvir/Velpatasvir/Voxilaprevir for 12 weeks as a salvage regimen in NA inhibitor-experienced patients with genotype 1-6 infection: the phase 3 POLARIS-1 study. Hepatology 2016;64(Suppl S1) [Abstract 194].

[41] Aragri M, Di Maio VC, Di Paolo D, et al. In the era of new direct acting antiviral agents HCV sequencing allows the most accurate subtype and genotype assignment. Journal of Hepatology 2016;64:S419-20.

[42] Di Maio VC, Cento V, Lenci I, et al. Multiclass HCV resistance to interferonfree direct acting antivirals regimens in real life failures advocates for tailored second-line therapies. Digestive and Liver Disease 2017;49S:e49-50 [F-14].

[43] Wyles D, Pockros P, Morelli G, et al. Ledipasvir-sofosbuvir plus ribavirin for patients with genotype 1 hepatitis $C$ virus previously treated in clinical trials of sofosbuvir regimens. Hepatology 2015;61:1793-7.

[44] Sullivan JC, De Meyer S, Bartels DJ, et al. Evolution of treatment-emergent resistant variants in telaprevir phase 3 clinical trials. Clinical Infectious Diseases 2013;57:221-9.

[45] Afdhal N, Reddy KR, Nelson DR, et al. Ledipasvir and sofosbuvir for previously treated HCV genotype 1 infection. New England Journal of Medicine 2014;370:1483-93.

[46] Dvory-Sobol H, Wyles D, Ouyang W, et al. Long-term persistence of HCV NS5A variants after treatment with NS5A inhibitor ledipasvir. Journal of Hepatology 2015;62:S221.

[47] Lahser F, Galloway A, Hwang P, et al. Interim analysis of a 3-year follow-up study of NA and NS3 resistance-associated variants (RAVs) after treatment with Grazoprevir-containing regimens in patients with chronic hepatitis $C$ virus (HCV) infection. Hepatology 2016;64(Suppl. S1) [Abstract 61].

[48] Wyles D, Dvory-Sobol H, Svarovskaia ES, et al. Post-treatment resistance analysis of hepatitis C virus from phase II and III clinical trials of ledipasvir/sofosbuvir. Journal of Hepatology 2016:30699-707 [Epub ahead of print].

[49] Poordad F, Gordon SC, Asatryan A, et al. High efficacy of ABT-493 and ABT-530 in HCV genotype 1-infected patients who have failed direct-acting antiviral-containing regimens: the MAGELLAN-1 study. Journal of Hepatology 2016;64:S160-1.

[50] Ceccherini Silberstein F, Di Maio VC, Aragri M, et al. Hepatitis C virus gene sequencing as a tool for precise genotyping in the era of new direct antiviral agents. Hepatology 2016;63:1058-9. 
[51] Kowdley KV, Gordon SC, Reddy KR, et al. Ledipasvir and sofosbuvir for 8 or 12 weeks for chronic HCV without cirrhosis. New England Journal of Medicine 2014;370:1879-88.

[52] Zeuzem S, Ghalib R, Reddy KR, et al. Grazoprevir-Elbasvir combination therapy for treatment-naive cirrhotic and non cirrhotic patients with chronic hepatitis $C$ virus genotype 1,4 , or 6 infection: a randomized trial. Annals of Internal Medicine 2015:163:1-13

[53] Kwo P, Gane E, Peng CY, et al. Efficacy and safety of grazoprevir/elbasvir plus or minus RBV for 12 weeks in patients with HCV G1 or G4 infection who previously failed peginterferon/RBV: C-EDGE Treatment-Experienced trial. Journal of Hepatology 2015:62:S674-5.

[54] Zeuzem S, Mizokami M, Planko S, et al. NS5A resistance-associated substitutions in patients with genotype 1 hepatitis $C$ virus: prevalence and effect on treatment outcome. Journal of Hepatology 2017 [Epub ahead of print].

[55] Sarrazin C, Dvory-Sobol H, Svarovskaia ES, et al. Prevalence of resistanceassociated substitutions in HCV NS5A, NS5B, or NS3 and outcomes of treatment with Ledipasvir and Sofosbuvir. Gastroenterology 2016;151:501-12.

[56] Desnoyer A, Pospai D, Lê MP, et al. Pharmacokinetics, safety and efficacy of a full dose sofosbuvir-based regimen given daily in hemodialysis patients with chronic hepatitis C. Journal of Hepatology 2016;65:40-7
[57] De Nicola S, Aghemo A. The quest for safe and effective treatments of chronic hepatitis $C$ in patients with kidney impairment. Liver International 2016;36:791-3.

[58] Saxena V, Koraishy FM, Sise ME, et al. Safety and efficacy of sofosbuvircontaining regimens in hepatitis C-infected patients with impaired renal function. Liver International 2016;36:807-16.

[59] Pockros PJ, Reddy KR, Mantry PS, et al. Efficacy of direct-acting antiviral combination for patients with hepatitis $C$ virus genotype 1 infection and severe renal impairment or end-stage renal disease. Gastroenterology 2016;150:1590-8.

[60] Gane EJ, Solà R, Cohen E, et al. RUBY-II: efficacy and safety of a ribavirin-free Ombitasvir/Paritaprevir/Ritonavir \pm Dasabuvir regimen in patients with severe renal impairment or end-stage renal disease and HCV genotypes 1a or 4 infection. Hepatology 2016;64(Suppl. S1) [Abstract 935].

[61] Roth D, Nelson DR, Bruchfeld A, et al. Grazoprevir plus elbasvir in treatmentnaive and treatment-experienced patients with hepatitis $C$ virus genotype 1 infection and stage 4-5 chronic kidney disease (the C-SURFER study): a combination phase 3 study. The Lancet 2015;386:1537-45.

[62] Craxi A, Perno CF, Viganò M, et al. From current status to optimization of HCV treatment: recommendations from an expert panel. Digestive and Liver Disease 2016;48:995-1005. 